\title{
El dibujo vectorial como recurso gráfico para la educación artística.
}

Vector drawing as a graphic resource for art education

\author{
Inmaculada Villagrán Arroyal \\ Universidad de Málaga \\ inmvillarr@uma.es
}

$\begin{array}{ll}\text { Recibido 15/09/2020 } & \text { Revisado 10/10/2020 } \\ \text { Aceptado 10/10/2020 } & \text { Publicado 31/10/2020 }\end{array}$

\section{Resumen:}

Las herramientas digitales para el dibujo son un recurso cada vez más extendido en la educación artística. Sin embargo, aún es difícil de encontrar estos medios gráficos como parte de la programación de muchas asignaturas de expresión artística o expresión gráfica en cursos de secundaria y bachillerato artístico, así como en asignaturas universitarias de temática gráfica.

Llevamos varios años investigando el porqué de esa carencia y para ello hemos ido desarrollando una serie de recursos virtuales que están sirviendo de apoyo al aprendizaje de estas herramientas digitales. El dibujo vectorial, entre otros, es un sistema digital alternativo a cualquier herramienta convencional o al método que ofrece un programa estándar digital. En el mundo profesional, el dibujo vectorial se reconoce como la tecnología más utilizada y funcional dentro del campo del diseño y la ilustración actual, pero también es una gran desconocida durante la enseñanza básica y en consecuencia, también en el momento de realizar los primeros estudios universitarios en Bellas Artes o Publicidad.

Sugerencias para citar este artículo,

Villagrán Arroyal, Inmaculada, (2020).El dibujo vectorial como recurso gráfico para la educación artística. Tercio Creciente (Monográfico extraordinario II), págs. 19-29, https://dx.doi.org/10.17561/rtc.extra2.5763

VILLAGRÁN ARROYAL, INMACULADA. El Dibujo vectorial como recurso gráfico para la educación artística. Tercio Creciente (Monográfico extraordinario II), octubre 2020, pp. 19-29, https://dx.doi.org/10.17561/rtc.extra2.5763 


\section{Abstract:}

Digital tools for drawing are an increasingly extended resource in art education. However, it is still difficult to find these graphic media as part of the programming of many subjects of artistic expression or graphic expression in high school and art baccalaureate courses, as well as in graphic-themed university subjects.

We have been investigating the reasons for this absence for several years and for this we have been developing a series of virtual resources that are supporting the learning of these digital tools. Vector drawing, among others, is an alternative digital system to any conventional tool or to the method offered by a standard digital program. In the professional world, vector drawing is recognized as the most used and functional technology in the field of design and current illustration, but it is also a great unknown during basic education and consequently, also at the time of carrying out the first studies university students in Fine Arts or Advertising.

Palabras Clave: Dibujo, Véctor, Ilustración, Diseño Gráfico, MOOC.

Key words: Drawing, Vector, Ilustration, Graphic Design, MOOC.

Sugerencias para citar este artículo,

Villagrán Arroyal, Inmaculada, (2020).El dibujo vectorial como recurso gráfico para la educación artística. Tercio Creciente (Monográfico extraordinario II), págs. 19-29, https://dx.doi.org/10.17561/rtc.extra2.5763

VILLAGRÁN ARROYAL, INMACULADA. El Dibujo vectorial como recurso gráfico para la educación artística. Tercio Creciente (Monográfico extraordinario II), octubre 2020, pp. 19-29, https://dx.doi.org/10.17561/rtc.extra2.5763 


\section{Descripción}

Con esta comunicación virtual queremos compartir la investigación y desarrollo de los contenidos básicos que se realizaron para el Proyecto de Innovación Educativa (PIE 17-195) de la Universidad de Málaga durante los cursos 2017-19, centrado en el análisis y estudio de los resultados obtenidos entre los estudiantes de primaria, secundaria y bachillerato artístico en relación al conocimiento y manejo de las herramientas vectoriales para el diseño e ilustración digital mediante el entorno de conocimiento abierto Open Course Ware (OCW) donde se diseñó una web de iniciación al aprendizaje de las herramientas vectoriales. Esta actividad fue el punto de arranque de nuestro actual Proyecto de Innovación e investigación (PIE 19-143) donde estamos desarrollando un MOOC (curso online masivo y abierto) enfocado a unir el conocimiento y la conectividad online.

Con nuestra aportación, pretendemos animar, orientar y concienciar de la necesidad urgente de promover esta tecnología. Nos encontramos cada día con nuevas formas y nuevas reinterpretaciones del lenguaje creativo y con nuestra propuesta intentamos llenar este vacío que tan a menudo encontramos dentro de las actuales programaciones. Dentro de la labor docente en la enseñanza artística, debemos combinar tanto los medios y técnicas tradicionales con la formación tecnológica, ya que serán herramientas imprescindibles para el futuro artista visual. Pretendemos por tanto, que el alumnado de Bellas Artes o Publicidad y principalmente los de nuevo ingreso, se familiaricen con el manejo de estas herramientas gráficas vectoriales realizando de manera autónoma un curso abierto en el que se les forme para su buen hacer técnico en futuros proyectos de diseño e ilustración.

Nuestra comunicación virtual muestra cómo este MOOC servirá como material de apoyo a las asignaturas gráficas que necesiten de soporte digital para su ejecución. La aplicación telemática estará abierta a toda la comunidad universitaria, pudiendo ser aprovechada por muchas otras titulaciones que precisen del conocimiento de estas técnicas digitales.

El dibujo vectorial, es un sistema digital alternativo a cualquier herramienta convencional o al método que ofrece un programa estándar basado en mapa de bits. En el mundo profesional, estos recursos se reconocen como la tecnología más utilizada y funcional dentro del campo del diseño gráfico y la ilustración, sobre todo por su peculiar forma de creación y composición de sus objetos basados en vectores, por la flexibilidad y versatilidad del manejo de sus trazos, de su peculiar sistema de construcción mediante nodos o anclas, de sus infinitas posibilidades de edición o de su salida de impresión a cualquier escala sin pérdida de calidad. 
ISSN: 2340-9096
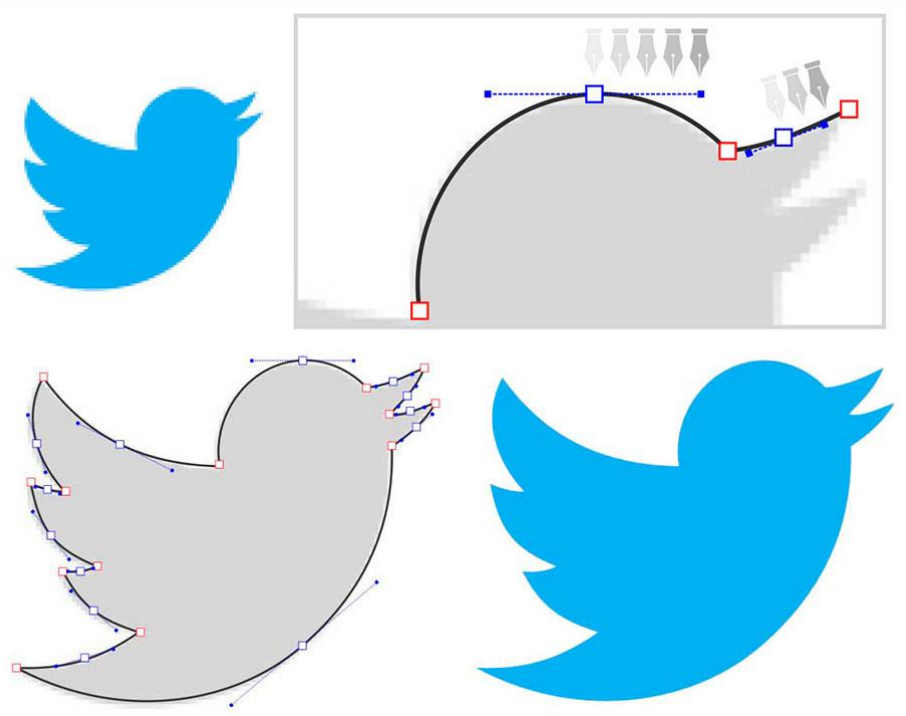

Imag. 01. Proceso de construcción de dibujo vectorial del símbolo de la red social Twitter. Ilustración de elaboración propia, 2016

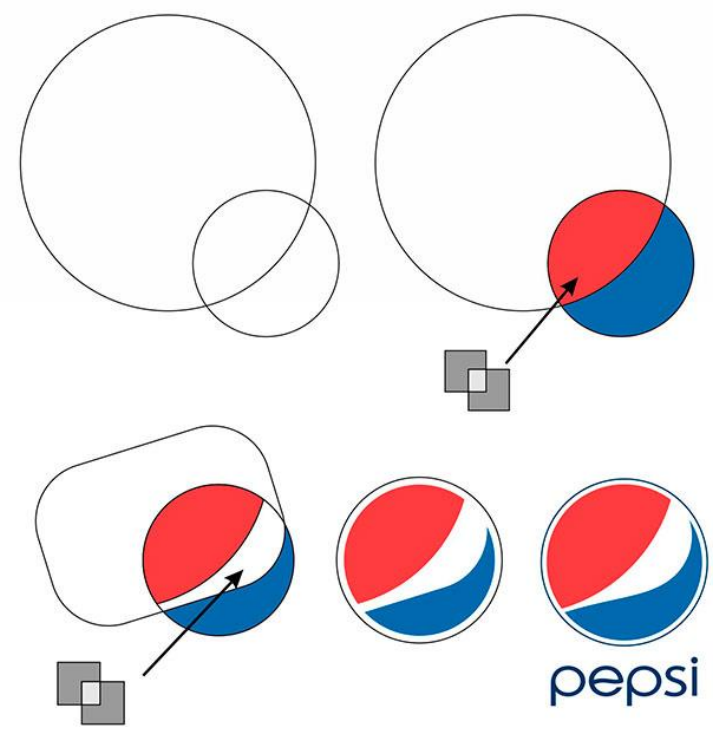

Imag. 02. Proceso de construcción de dibujo vectorial del símbolo de la marca pepsi. Ilustración de elaboración propia, 2016 
ISSN: $2340-9096$

https://dx.doi.org/10.17561/rtc.extra2.5763

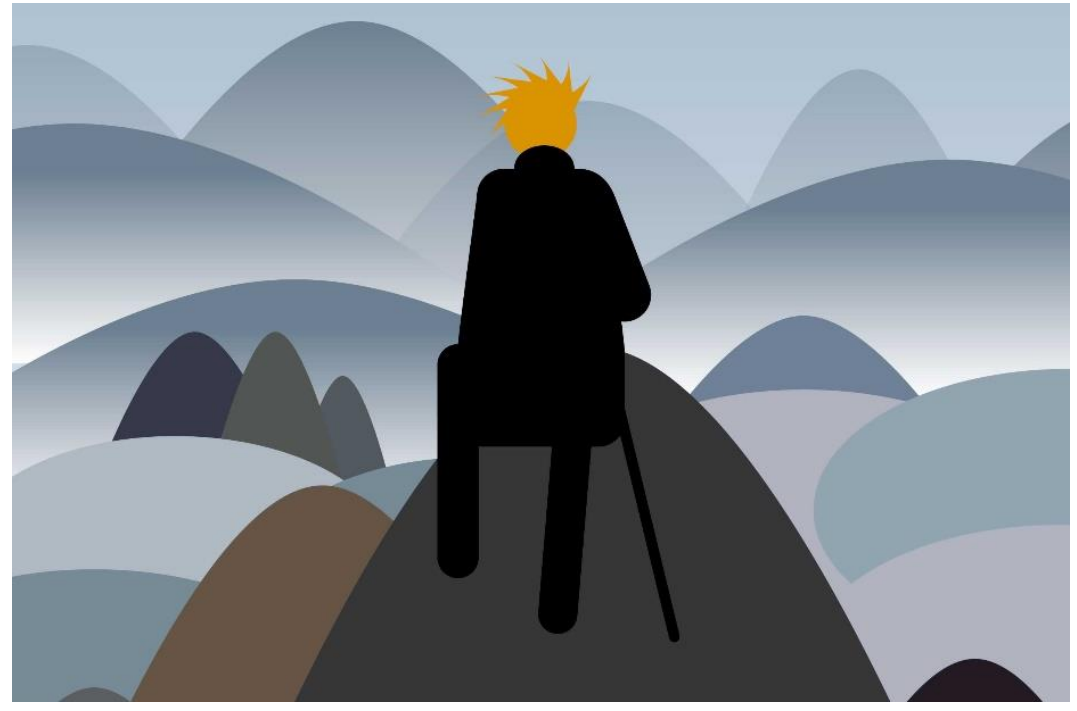

Imag. 03. Interpretación vectorial del cuadro El caminante sobre el mar de nubes de C.D. Friedrich. Ilustración de elaboración propia, 2019

La aparición de las herramientas gráficas basadas en vectores a principios de los 90 , fueron desde sus inicios una solución crucial en la representación y composición para todos los dibujantes con inquietudes tecnológicas y actualmente debería ser ya parte del aprendizaje técnico en cualquier asignatura de dibujo o expresión artística desde los primeros cursos de primaria, secundaria o bachillerato. 
ISSN: $2340-9096$

https://dx.doi.org/10.17561/rtc.extra2.5763

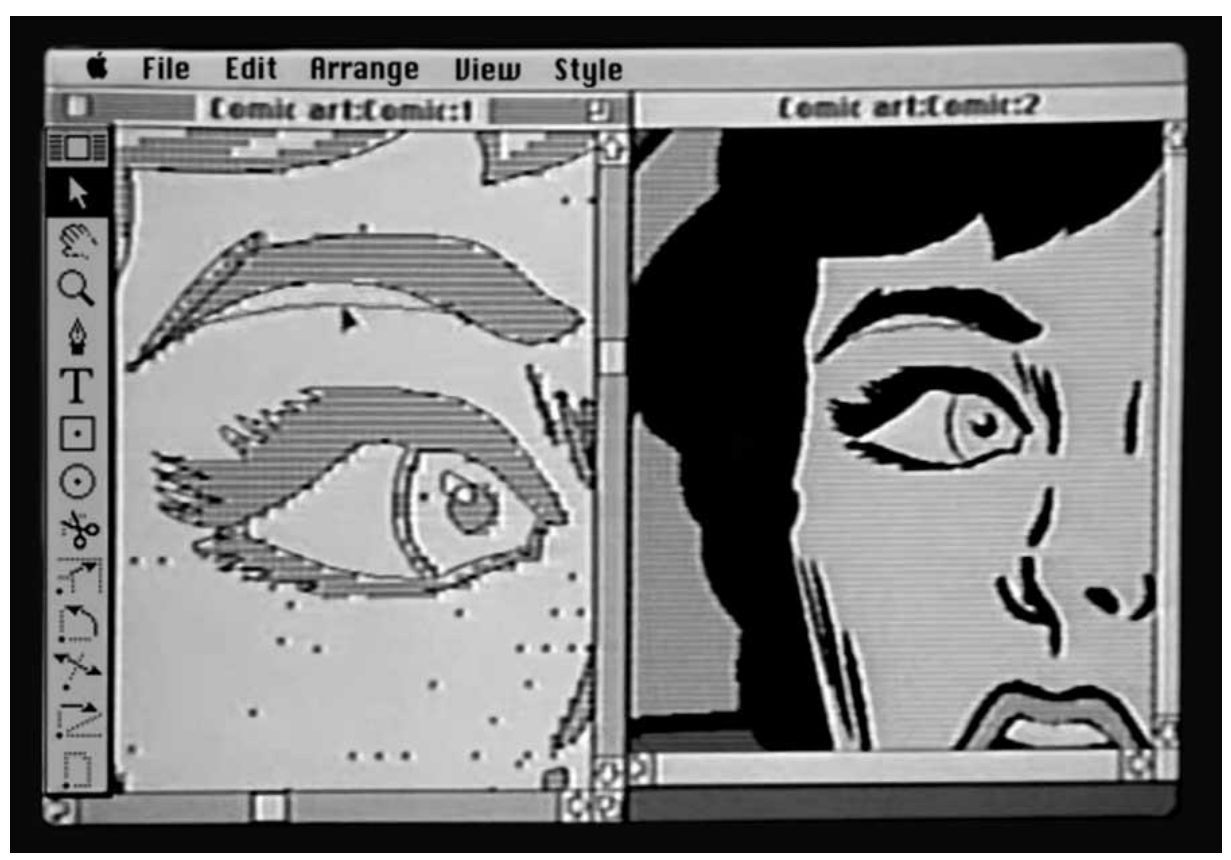

Imag. 04. Fotograma del vídeo de presentación de Illustrator 1.1 por John Warnock en 1987 mostrando el funcionamiento de las herramientas vectoriales. Meet Adobe Illustrator. https://youtu.be/oeIbkxOTj04

Desde 2017, llevamos varios años investigando para nuestro Proyecto de Innovación Educativa de la Universidad de Málaga, el porqué de esa carencia docente y para ello hemos desarrollado una serie de recursos virtuales que están sirviendo de apoyo al aprendizaje de estas herramientas digitales. Por tanto, con esta comunicación virtual queremos compartir la investigación y desarrollo de los contenidos básicos que hemos ido realizando en relación al conocimiento y manejo de las herramientas vectoriales para el diseño y la ilustración digital. 
ISSN: 2340-9096

https://dx.doi.org/10.17561/rtc.extra2.5763

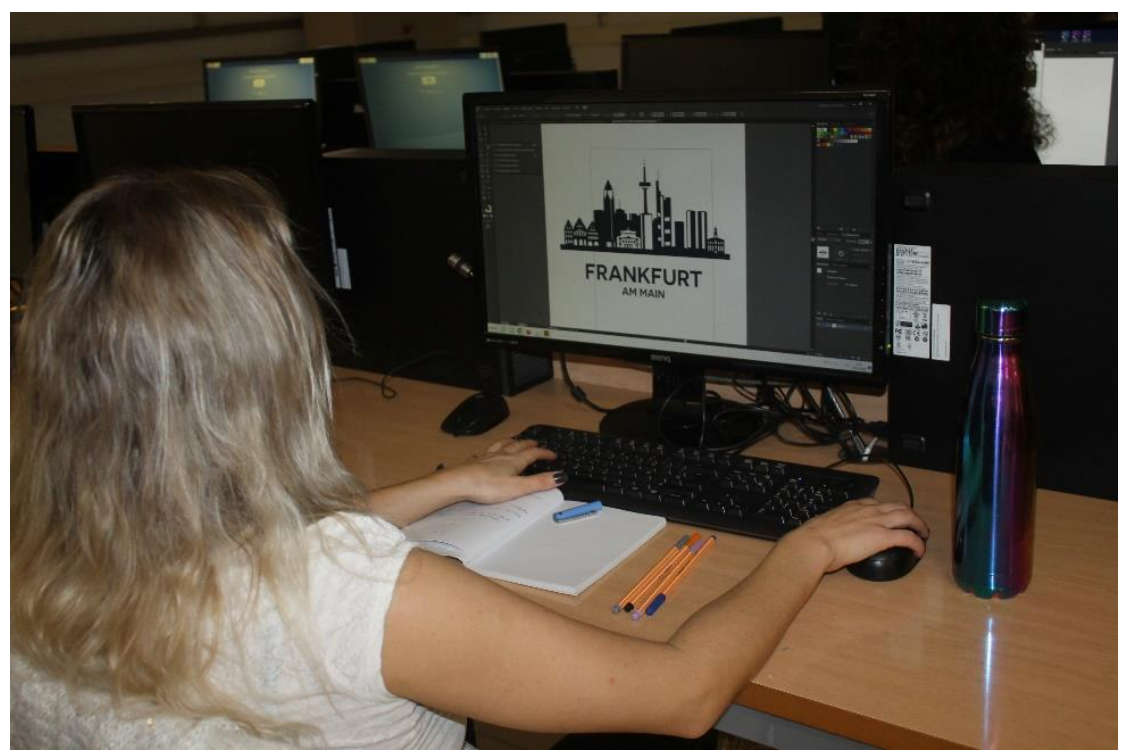

Imag. 05. Alumnos/as del IV Curso de extensión universitaria en técnicas gráficas vectoriales para la ilustración con Adobe Illustrator, Facultad de Bellas Artes de Málaga 2019-20. Fotografía propia.

Este proyecto ha estado centrado en el análisis y estudio de unos resultados obtenidos entre los/as estudiantes de primaria, secundaria y bachillerato artístico mediante un entorno de conocimiento abierto, donde se diseñó una web de iniciación al aprendizaje de las herramientas vectoriales.

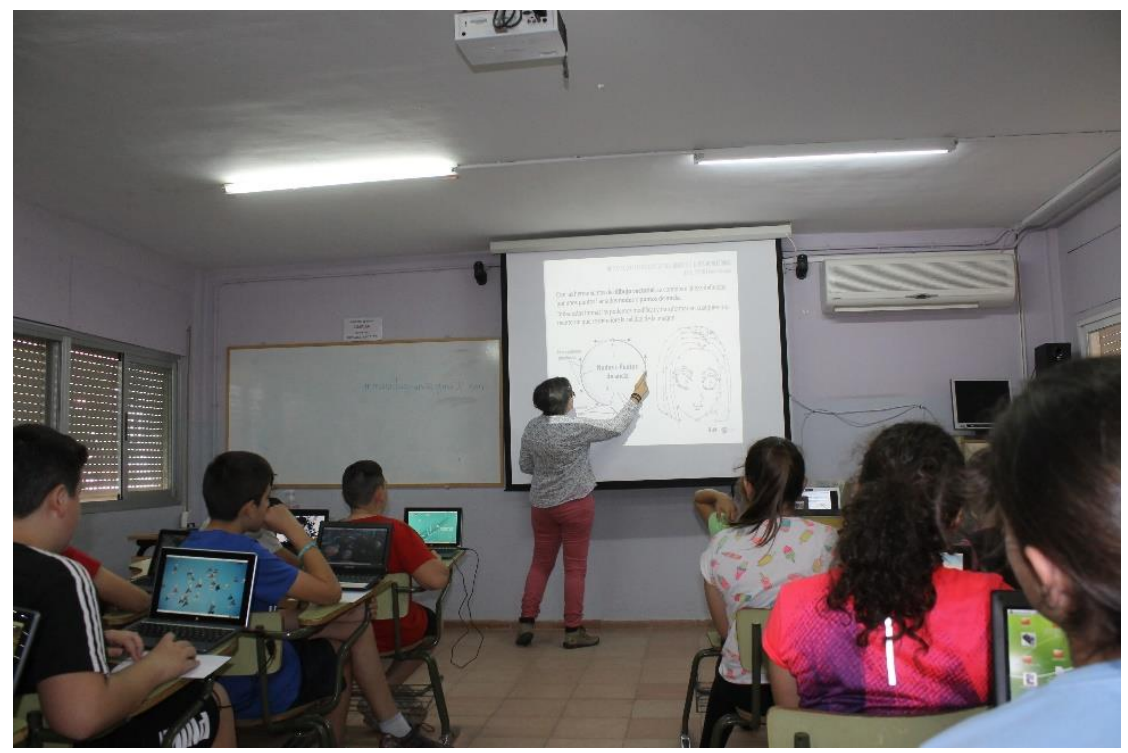

Imag. 06. Taller de dibujo vectorial, $6^{\circ}$ de primaria, Colegio El Retiro, Jerez de la Fra, 2018.Fotografía propia. 
ISSN: 2340-9096

https://dx.doi.org/10.17561/rtc.extra2.5763

Esta actividad fue el punto de arranque de nuestro Proyecto de Innovación Educativa donde actualmente nos hemos embarcado. Se trata del desarrollo de un MOOC o más conocido como Curso Online Abierto que está enfocado a unir el conocimiento y la conectividad online mediante videotutoriales didácticos y donde toda la comunidad universitaria puede seguir los temas organizando su propio ritmo de aprendizaje. Con nuestra aportación, pretendemos animar, orientar y concienciar de la necesidad de promover esta tecnología digital, tanto a docentes como a toda la comunidad de estudiantes afines al diseño y la ilustración contemporánea y así facilitarles un buen hacer para todo tipo de proyectos gráficos.

$\mathrm{Al}$ iniciar nuestra investigación, realizamos diferentes talleres en Colegios de Enseñanza Primaria para ver el actual nivel de manejo gráfico digital entre alumnos y alumnas de entre 11 y 12 años y pudimos comprobar en esos pequeños nativos digitales, el rápido aprendizaje de estas herramientas a base de curvas.

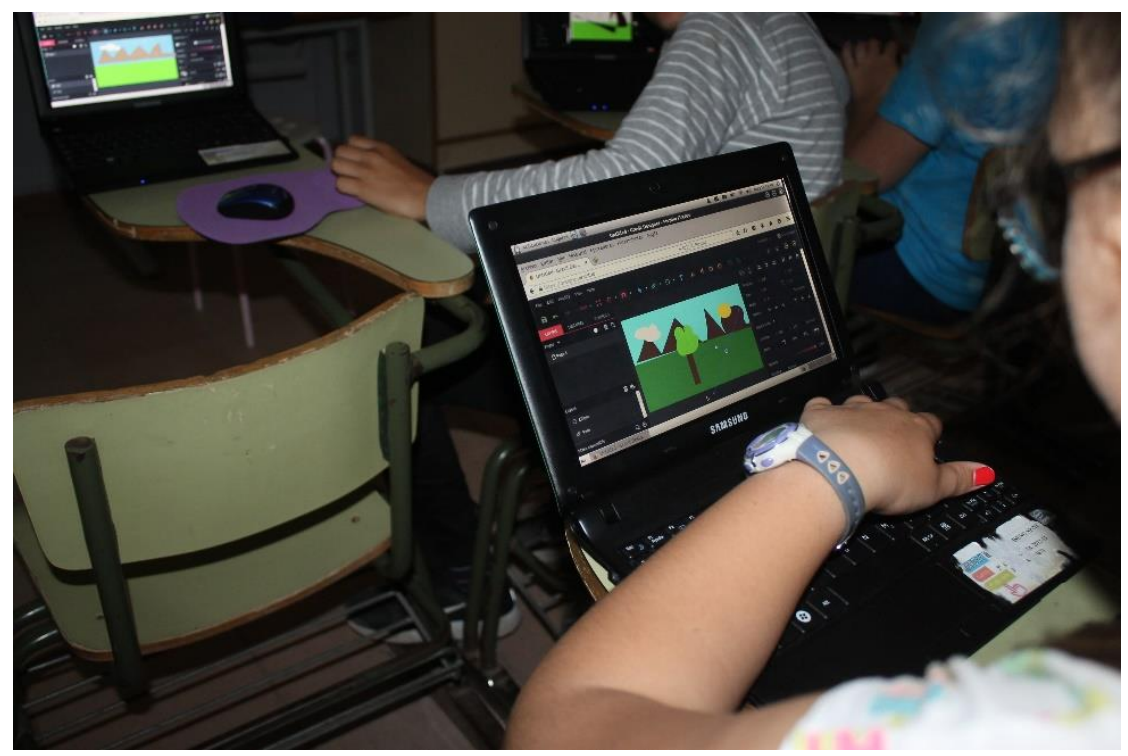

Imag. 07. Taller de dibujo vectorial, $6^{\circ}$ de primaria, Colegio El Retiro, Jerez de la Frontera, 2018. Fotografía propia. 
ISSN: $2340-9096$

https://dx.doi.org/10.17561/rtc.extra2.5763

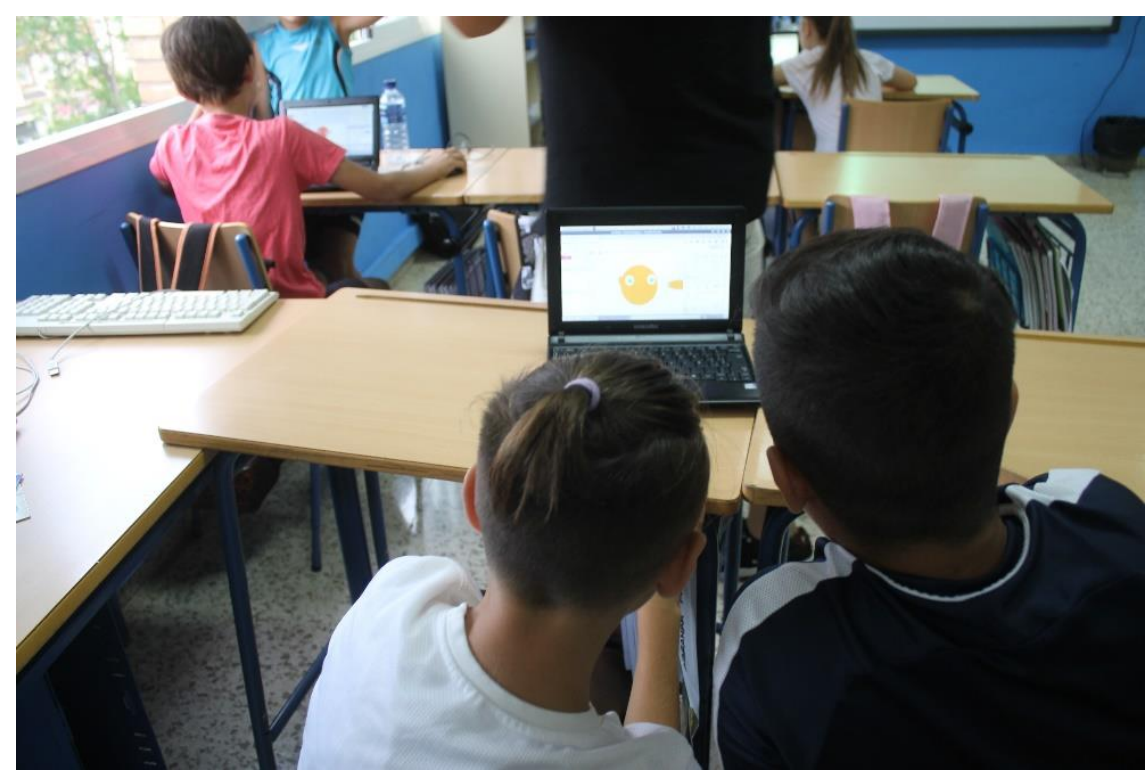

Imag. 08. Taller de dibujo vectorial, $6^{\circ}$ de primaria, Colegio Bergamín, Málaga, 2018. Fotografía propia.

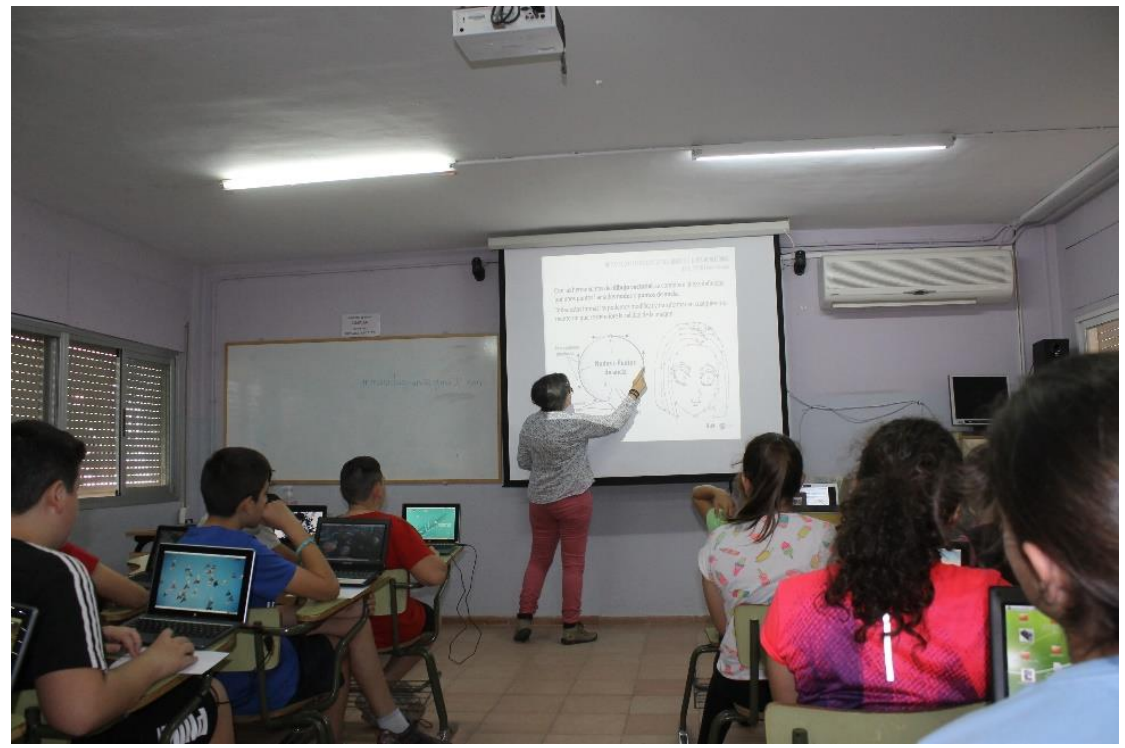

Imag. 09. Taller de dibujo vectorial, $6^{\circ}$ de primaria, Colegio Bergamín, Málaga, 2018. Fotografía propia.

Sin embargo, durante nuestras charlas con diferentes profesores de las asignaturas artísticas de cada centro, ninguno contemplaba estas herramientas de dibujo como parte de las unidades didácticas. Siendo en ocasiones incluso la primera vez que oían hablar de ellas. 
Nos encontramos cada día con nuevas formas y nuevas reinterpretaciones del lenguaje creativo y con nuestra propuesta intentamos llenar este vacío que tan a menudo encontramos dentro de las actuales programaciones. Dentro de la labor docente en la enseñanza artística, creemos que debemos combinar tanto los medios y técnicas tradicionales como la formación tecnológica, ya que serán herramientas imprescindibles para el futuro artista visual.

Así pues, pretendemos, que el alumnado de Bellas Artes o Publicidad y principalmente los de nuevo ingreso, se familiaricen con el manejo de estas herramientas gráficas y más aún si realizan de manera autónoma el curso abierto que les pueda formar técnicamente para sus futuros proyectos de diseño e ilustración.

Este MOOC lo construimos todo un equipo de profesionales del diseño, la ilustración y la docencia y esperamos que pronto sea un material de apoyo a las asignaturas gráficas que necesitan de soporte digital para su ejecución. Por último decir que esta aplicación telemática estará abierta a todas las comunidades universitarias, pudiendo ser aprovechada por todas las titulaciones que precisen de este conocimiento.

\section{Acceso a la comunicación virtual presentada en CIVARTES}

https://youtu.be/AgtfuEBP6ZM

\section{Referencias}

ALCALÁ, J.R. La piel de la imagen. Ensayos sobre gráfica en la cultura general, Sendemá, Valencia 2011

BOUNFORD, T. Diagramas digitales. Cómo diseñar y presentar información gráfica. Gustavo Gili, Barcelona 2011.

COLLIN, S. Diccionario de Multimedia, Mac Graw Hill/Interamericana, Santafé de Bogotá 1996.

DANS, E. Todo va a cambiar. Tecnología y evolución: adaptarse o desaparecer, Deusto, Barcelona 2010.

GASCH, M. Curso Práctico de Diseño Gráfico por Ordenador: Conceptos Generales, Génesis, Madrid 1991.

GÓMEZ MOLINA, J.J. Máquinas y herramientas de dibujo, Cátedra, Madrid 2002. 
JOHANSSON, K., LUNDBERG, P. y RYBERG, R. Manual de producción gráfica. Recetas, Gustavo Gili, Barcelona 2011.

KLINE, M. Matemáticas en el mundo moderno. Versión española de GUZMÁN OZAMIZ, M. Blume, Madrid 1974.

LARDNER, J y ROBERTS, P. Técnicas de arte digital para ilustradores y artistas, Acanto, Barcelona, 2012.

LÓPEZ LORENTE, F.C. Ilustración y diseño con ordenador, Ra-ma, Madrid 1992.

MANOVICH, L. El lenguaje de los nuevos medios de comunicación, Paidós, Barcelona 2005.

MANOVICH, L. El software toma el mando, UOCpress, Barcelona 2013.

ORIHUELA, J.L. y SANTOS, M.L. Introducción al diseño digital. Concepción y Desarrollo de Proyectos de Comunicación Interactiva, Anaya Multimedia, Madrid 1999.

SATUÉ, E. El diseño gráfico desde los orígenes hasta nuestros días, Alianza Forma, Madrid 1999.

SEDDON, T. Imágenes. Flujo de trabajo digital para diseñadores gráficos, Gustavo Gili, Barcelona 2008.

SIMPSON, I. La nueva guía de la ilustración, Blume, Barcelona 1993.

SPARKE, P. Diseño y Cultura. Una Introducción, Gustavo Gili, Barcelona 2010.

VILLAGRÁN ARROYAL, I. Creación de Elementos Gráficos. IC Editorial, Antequera, Málaga. 2014.

VITTA, M. El sistema de las imágenes. Estética de las representaciones cotidianas, Paidós, Barcelona 2003.

WONG, W y WONG, B. Diseño gráfico digital. Gustavo Gili, Barcelona 2004. 
Revista de Estudios en Sociedad Artes y Gestión Cultural

ISSN: 2340-9096

https://dx.doi.org/10.17561/rtc.extra2.5763
Monográfico

30

Extraordinario I

Octubre 2020

Investigación 